\title{
Semiologia na unidade de terapia intensiva: qualificando a assistência
}

\author{
Semiology in the intensive care unit: qualifying assistance
}

$\mathrm{N}$ os últimos anos, o número de indivíduos que necessitam de internações hospitalares tem aumentado de forma alarmante. No Brasil, somente no ano de 2016, foram contabilizadas pelo Sistema Único de Saúde Pública (SUS) mais de 11 milhões de internações hospitalares, sendo mais de 800 mil de alta complexidade1. Sabe-se que os danos clínicos causados pela doença de base, somados ao tempo de permanência em unidades de cuidado intensivo, acarretam a esses pacientes complicações de ordem mental, psicológica e, sobretudo, funcional. Estamos diante, portanto, da premissa básica do cuidado multiprofissional, da importância da busca ativa por instrumentos de avaliação e detecção precoce de distúrbios limitantes, que impactam desde a alta da unidade, alta hospitalar até retomada às atividades diárias ${ }^{2,3}$.

Muitas vezes, a própria terapia curativa acaba por limitar a mobilização desses pacientes, que podem necessitar tempo maior de ventilação mecânica invasiva, com consequente sedoanalgesia, uso de corticoesteroides, instabilidade hemodinâmica com necessidade de drogas vasoativas, e múltiplas vias de acesso, o que culmina em complicações inerentes à restrição ao leito. O efeito cascata se instala levando à imobilidade, aparecimento de sarcopenia, osteopenia, encurtamento muscular e mais limitações².

No contexto de prevenção e reabilitação, a fisioterapia atua como aliada afim de minimizar os prejuízos da imobilidade, auxiliando na redução da dor, evitando e/ou abreviando os danos do hipotrofismo muscular ventilatório e periférico, além de contribuir para o progresso das funções físicas e respiratórias ${ }^{4,5}$.

Muito se estuda a respeito de ferramentas úteis na detecção desses danos. Diversas escalas se propõem a avaliar aspectos funcionais de pacientes internados em Unidades de Terapia Intensiva (UTI) e, dentre essas, pode-se destacar a desenvolvida por Perme et al. ${ }^{6}$. Tal escore é específico para mensurar a melhora da condição de mobilidade, levando em consideração fatores externos limitantes, de forma a se padronizar a avaliação do paciente na UTI e qualificar a assistência multiprofissional ${ }^{6}$.

Em nossa realidade, a classificação funcional obtida por meio dessa ferramenta, associada à expertise dos fisioterapeutas, propiciam a elaboração de um plano terapêutico específico e voltado às limitações funcionais de cada paciente, tornando o cuidado altamente específico e baseado em evidência e avaliação prática.

Cabe a nós, profissionais da saúde, a preocupação com a prevenção e reabilitação precoce de nossos pacientes tendo como base sólida a semiologia, que mesmo com o avanço das técnicas terapêuticas, permanece soberana e norteadora.

Clarissa Netto Blattner Fisioterapeuta. Doutora em Ciências Cardiovasculares - Cardiologia (UFRGS). Professora do Curso de Fisioterapia da FAENFI/PUCRS. 


\section{REFERÊNCIAS}

1. Brasil. Ministério da Saúde. Sistema de Informações Hospitalares do SUS (SIH/SUS). Disponível em: http://tabnet.datasus.gov. br/cgi/tabcgi.exe?sih/cnv/sxuf.def

2. Hodgson CL, Udy AA, Bailey M, Barrett J, Bellomo R, Bucknall T, Gabbe BJ, Higgins AM, Iwashyna TJ, Hunt-Smith J, Murray LJ, Myles PS, Ponsford J, Pilcher D, Walker C, Young M, Cooper DJ. The impact of disability in survivors of critical illness. Intensive Care Med. 2017;43(7):992-1001. https://doi.org/10.1007/s00134-017-4830-0

3. Muscedere J, Waters B, Varambally A, Bagshaw SM, Boyd JG, Maslove D, Sibley S, Rockwood K. The impact of frailty on intensive care unit outcomes: a systematic review and meta-analysis. Intensive Care Med. 2017;43(8):1105-22. https://doi.org/10.1007/ s00134-017-4867-0

4. Martinez BP, Batista AKMS, Gomes IB, Olivieri FM, Camelier FW, Camelier AA, Frequency of sarcopenia and associated factors among hospitalized elderly patients. BMC Musculoskelet Disord. 2015;16:108. https://doi.org/10.1186/s12891-015-0570-x

5. Jesus FS, Paim D de M, Brito J de O, Barros I de A, Nogueira TB, Martinez BP, Pires TQ. Mobility decline in patients hospitalized in an intensive care unit. Rev Bras Ter Intensiva. 2016;28(2):114-9. https://doi.org/10.5935/0103-507X.20160025

6. Perme C, Nawa RK, Winkelman C, Masud F. A tool to assess mobility status in critically ill patients: The Perme Intensive Care Unit Mobility Score. Methodist Debakey Cardiovasc J. 2014;10(1):41-9. https://doi.org/10.14797/mdcj-10-1-41 